\title{
Accuracy Aspects in Multiphase Flow Metering Using X-Ray Transmission
}

\author{
Victor R. Bom, Martijn C. Clarijs, Carel W. E. van Eijk, Zvonimir I. Kolar, Joop Frieling, Lex M. Scheers, and \\ Gary J. Miller
}

\begin{abstract}
The composition of a mixture of several components flowing through a pipe can be determined by means of an X-ray transmission technique/analysis. We present some aspects of the theory relating to an application in which the component volume fractions of an oil, water, and gas mixture are determined. It will be shown that on fundamental grounds, this technique has its limits regarding the number of components that can be distinguished in relation to the achieved accuracy of the measured attenuation.
\end{abstract}

Index Terms-Multiphase flow, X-ray transmission.

\section{INTRODUCTION}

$\mathbf{I}$ N MANY fields of technology, including the oil industry, the need exists to probe the content of pipes, preferably with a noninvasive technique. A suitable method of nondestructive testing for this purpose is based on X-ray transmission [1], [2].

The method comprises the measurement of the attenuation by the pipe including its contents at a number of suitable photon energies. A system of equations can then be constructed in which the component characteristics determine the coefficients and the component abundances or fractions are the unknowns. Every energy yields one equation, and in addition there is the condition of the pipe's being filled completely. This system of equations can be solved for the component fractions. Operational systems based on this technique exist, for instance, for the analysis of oil, water, and gas mixtures, which come from oil wells. A problem in this case is formed by the variation of the salinity of the water component. Erroneous component fractions may result because of the uncertainty in the salinity in combination with the high absorption of salt. It appears that the method can be easily extended to measure the salt concentration by including an extra $\mathrm{X}$-ray energy. This would give rise to an additional equation and to an extra analyzable fraction [3].

In this paper, we show that the number of components that can be resolved does not simply depend on the number of energies that are used, but primarily on the number of physical absorption processes that are involved. This reduces the maximum

Manuscript received November 8, 2000; revised April 26, 2001.

V. R. Bom, M. C. Clarijs, and C. W. E. van Eijk are with the Radiation Technology Group, Interfaculty Reactor Institute, Delft University of Technology, 2629JB Delft, The Netherlands (e-mail: vb@iri.tudelft.nl).

Z. I. Kolar is with the Department of Radiochemistry, Interfaculty Reactor Institute, Delft University of Technology, 2629JB Delft, The Netherlands (e-mail kolar@iri.tudelft.nl).

J. Frieling and L. M. Scheers are with Shell International Exploration and Production B.V., 2280 AB Rijswijk, The Netherlands (e-mail: a.m.scheers@siep.shell.com).

G. J. Miller is with Daniel Europe Ltd., Stirlingshire, FK5 3NS Scotland, U.K. (e-mail: Gary_Miller@Danielind.co.uk).

Publisher Item Identifier S 0018-9499(01)10699-4.

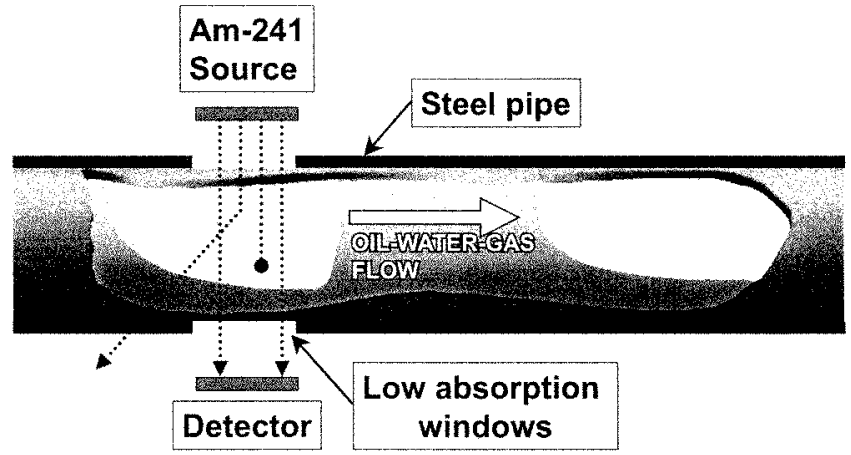

Fig. 1. Schematic layout of a multiphase flow meter using X-ray transmission.

number of distinguishable components basically to three. We assume that the energy dependence of the absorption processes is identical for the components involved. This is a very good approximation in the case of the crude oil application. If the minor differences between the shapes of the energy dependencies of the attenuation coefficients of the components are employed, a fourth component could be resolved, but this would require the measurement of the attenuation with extreme precision.

It is certainly true that the materials in the pipe completely determine the X-ray absorption. However, this does not infer the inverse statement.

\section{SETUP}

A schematic drawing of a typical setup used in the oil industry is shown in Fig. 1. The radiation beam is directed perpendicular to and through the center of a pipe carrying the flow of the oil, water, and gas mixture.

To measure the attenuation, a narrow beam is used in order to reduce the influence of scattering effects. Analysis of the measured attenuations yields the composition of the mixture within the beam area. Only for a homogeneous mixture will this result characterize the overall composition. Therefore, the setup may be employed after a "mixer device" to unsure homogeneity. Since the mixture composition can change rapidly depending on the flow regime, short measuring times of less than a second are normally used. The combination of the analyses of the many consecutive short measurements gives the desired average composition.

In commercial equipment, radioactive sources are used to obtain low-energy gamma or X-rays, but in principle $\mathrm{X}$-ray tubes can also be used. The radiation penetrates the steel pipe walls of the setup through high transmission windows. 
In view of the attainable accuracy, for a given radiation energy, the path length is a compromise between 1) a large absorption effect, which requires a large path length, and 2) the count rate in the detector, which benefits from a small path length. Path lengths of a few centimeters are most often applied. But also the radiation energy is a compromise [4], notably between 1) a large penetration, which requires a high energy, and 2) a large difference in absorption between oil and water, which requires a low energy. In oil-industry applications, this generally lies between 30 and $60 \mathrm{keV}$.

\section{X-RAY ABSORPTION}

The absorption of photons by matter is described by

$$
I=I_{0} e^{-\mu t}
$$

where $I$ and $I_{0}$ are the radiation intensities after and before absorption respectively, $\mu$ is the linear attenuation coefficient, and $t$ is the thickness of the absorber along the beam path. $\mu$ depends on the specific material properties and on the photon energy $E$.

In the case of absorption by a multiphase flow consisting of oil, water, and gas, (1) expands into

$$
I=I_{0} e^{\sum-\mu_{i}\left(\rho_{i}, E\right) t_{i}}, \quad i=1, \ldots, n
$$

where index $i$ indicates the components mentioned.

\section{A. Linear Attenuation Coefficient}

In the X-ray energy region, only the photoelectric effect and the Compton effect play a significant role in the absorption. The Rayleigh effect can be ignored because it never contributes more than $10 \%$

$$
\mu=\mu_{\text {Compton }}+\mu_{\text {photo }}
$$

From Fig. 2, it is evident that the energy dependence of these effects is functionally very nearly the same for all materials involved. Where the Compton effect is concerned, this can also be understood from a physical point of view. Since only low $Z$ materials are involved, the binding energy of even the K-shell electrons is low compared to the X-ray energies used. The electrons can therefore be considered unbound, and the atomic number will influence the magnitude of the effect, but not the energy dependence.

Under this condition, we can state a parameterized expression for $\mu$

$$
\mu=C_{\mathrm{p}} \pi(E)+C_{\mathrm{C}} \gamma(E)
$$

where the energy dependences of photo- and Compton effect are described by the atomic number independent functions $\pi(E)$ and $\gamma(E)$ with magnitudes $C_{\mathrm{p}}$ and $C_{\mathrm{C}}$, respectively. $C_{\mathrm{p}}$ and $C_{\mathrm{C}}$ depend on density and atomic number, but not on energy. More specifically, we have

$$
\begin{aligned}
\mu_{\mathrm{o}} & =C_{\mathrm{p}, \mathrm{o}} \pi(E)+C_{\mathrm{C}, \mathrm{o}} \gamma(E) \\
\mu_{\mathrm{H}_{2} \mathrm{O}} & =C_{\mathrm{p}, \mathrm{H}_{2} \mathrm{O}} \pi(E)+C_{\mathrm{C}, \mathrm{H}_{2} \mathrm{O}} \gamma(E) \quad \text { etc. }
\end{aligned}
$$

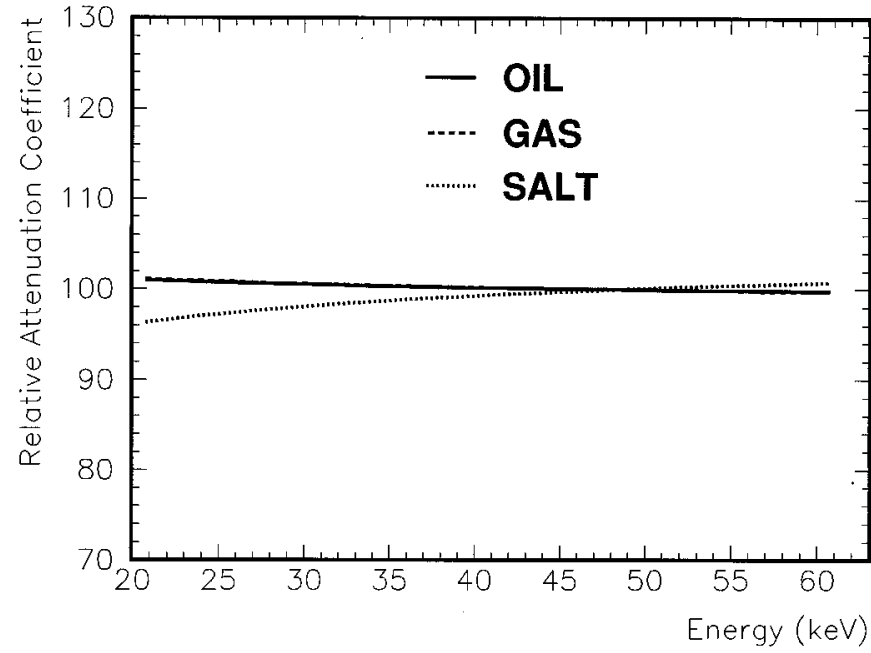

Fig. 2. Linear attenuation coefficients in various materials [5] due to the photoeffect (diagonal curves) and the Compton effect (horizontal-like curves). Oil: C-H mixture with $15 \%$ (weight) $\mathrm{H}$; Salt: $\mathrm{NaCl}$.

for oil, water, etc.

1) Saline Water: Water is often one of the components in the mixture that flows from oil wells. It is either present in the reservoir or artificially injected for the purpose of improving the oil extraction from the field. This water always contains an amount of salt, either dissolved from the underground rocks or present in the injected water. Usually this is $\mathrm{NaCl}$. The salt concentration in production water of water-injected reservoirs can change over time, and wells from different reservoir blocks can have different salinity. The density of the saline water increases linearly with the salt concentration

$$
\rho_{\mathrm{sw}}=\rho_{\mathrm{H}_{2} \mathrm{O}}+R d \rho_{\mathrm{s}}
$$

Here, "sw" stands for saline water, "s" for salt, and $\mathrm{H}_{2} \mathrm{O}$ for clear water; $d \rho_{\mathrm{s}}$ is the partial density of salt in the saline water, also called the salinity; $\rho_{\mathrm{H}_{2} \mathrm{O}}$ is the density of the clear water. The rate of increase $R$ varies in the range [0.6-0.9], depending on the kind of salt.

The attenuation coefficient of saline water is not so straightforward

$$
\mu_{\mathrm{sw}}=\mu_{\mathrm{H}_{2} \mathrm{O}}+d \rho_{\mathrm{s}}\left[\Lambda_{\mathrm{s}}-(1-R) \Lambda_{\mathrm{H}_{2} \mathrm{O}}\right]
$$

Here $\Lambda$ is the mass attenuation coefficient

$$
\Lambda_{i}(E)=\left(\frac{\mu}{\rho}\right)_{i} \text { for } i=\mathrm{s} \text { or } \mathrm{H}_{2} \mathrm{O} .
$$

This coefficient is independent of the density.

\section{ANALYSIS}

By measuring $I$ at $m$ different energies, one obtains as many equations

$$
-\ln \left(\frac{I_{j}}{I_{0}}\right)=\sum_{i=1}^{n} \mu_{i}\left(\rho_{i}, E_{j}\right) t_{i} \text { for } j=1,2, \ldots, m .
$$


Here $n$ is the number of mixture components considered. For nonmixing components, assuming a completely filled pipe of diameter $D$

$$
\sum_{i=1}^{n} t_{i}=D
$$

As the values for $\mu_{i}$ are "known," the set of equations can in principle be solved for the $t_{i} \mathrm{~s}$.

The absorber thicknesses $t_{i}$ are strictly valid only in the area of the radiation beam. Assuming a homogeneous mixture throughout the pipe volume, the mixture composition is derived from

$$
\alpha_{i}=\frac{t_{i}}{D}
$$

with $\alpha_{i}$ the mixture volume fraction of component $i$.

\section{A. Using Parameterized Attenuation Coefficients}

Substitution of (4) into (9) gives, after some algebraic exercise using (10)

$$
\text { attenuation }_{j}=-\ln \left(\frac{I_{j}}{I_{0}}\right)=P \pi\left(E_{j}\right)+Q \gamma\left(E_{j}\right) .
$$

Here $P$ and $Q$ are elaborate expressions containing only "known" terms such as the $C_{\mathrm{p}}$ and $C_{\mathrm{C}}$ coefficients, densities, etc.

$$
\begin{aligned}
& P=U t_{\mathrm{o}}+\left(V_{1}+V_{2} d \rho_{\mathrm{s}}\right) t_{\mathrm{sw}}+W t_{\mathrm{g}} \\
& Q=X t_{\mathrm{o}}+\left(Y_{1}+Y_{2} d \rho_{\mathrm{s}}\right) t_{\mathrm{sw}}+Z t_{\mathrm{g}} \\
& D=t_{\mathrm{o}}+t_{\mathrm{sw}}+t_{\mathrm{g}}
\end{aligned}
$$

with

$$
\begin{aligned}
U & =C_{\mathrm{p}, \mathrm{o}} \quad V_{1}=C_{\mathrm{p}, \mathrm{H}_{2} \mathrm{O}} \\
V_{2} & =\frac{C_{\mathrm{p}, \mathrm{s}}}{\rho_{\mathrm{s}}}-(1-R) \frac{C_{\mathrm{p}, \mathrm{H}_{2} \mathrm{O}}}{\rho_{\mathrm{H}_{2} \mathrm{O}}} \quad W=C_{\mathrm{p}, \mathrm{g}} \\
X & =C_{\mathrm{C}, \mathrm{o}} \quad Y_{1}=C_{\mathrm{C}, \mathrm{H}_{2} \mathrm{O}} \\
Y_{2} & =\frac{C_{\mathrm{C}, \mathrm{s}}}{\rho_{\mathrm{s}}}-(1-R) \frac{C_{\mathrm{C}, \mathrm{H}_{2} \mathrm{O}}}{\rho_{\mathrm{H}_{2} \mathrm{O}}} Z=C_{\mathrm{C}, \mathrm{g}} .
\end{aligned}
$$

Equation (12) describes the absorption by the mixture in the pipe as a function of energy. The first term $P$ relates to the photoeffect, while the second term $Q$ relates to the Compton effect. From this system, $P$ and $Q$ are resolved and substituted into equation system (13). This latter system only has three equations, allowing for three variables to be determined. These could be three absorber thicknesses $t_{i}$ but also, for example, the salinity and two absorber thicknesses.

The number of relevant parameters that can be determined from X-ray absorption is thus related to the number of underlying physical processes, and not to the number of energies $E_{j}$ at which the attenuation is measured. This holds true irrespective of the mixture homogeneity and of the flow regime, since these assumptions are not used in the above derivation. Homogeneity comes into play only when the $t_{i} \mathrm{~s}$ are translated into volume fractions for the total mixture (11).

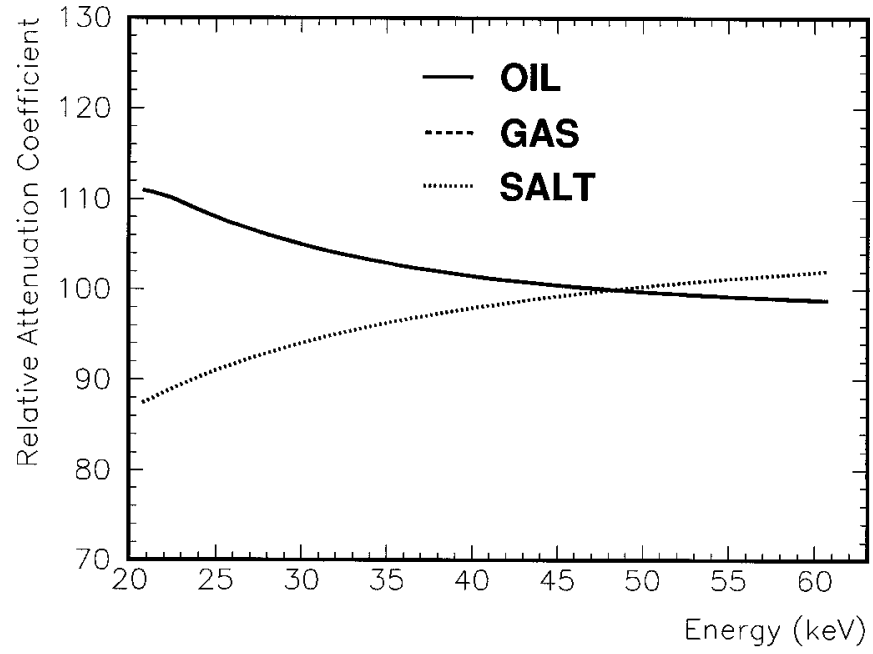

Fig. 3. Linear attenuation coefficients due to photoeffect, relative to the water attenuation coefficient, and normalized to $100 \%$ at $50 \mathrm{keV}$.

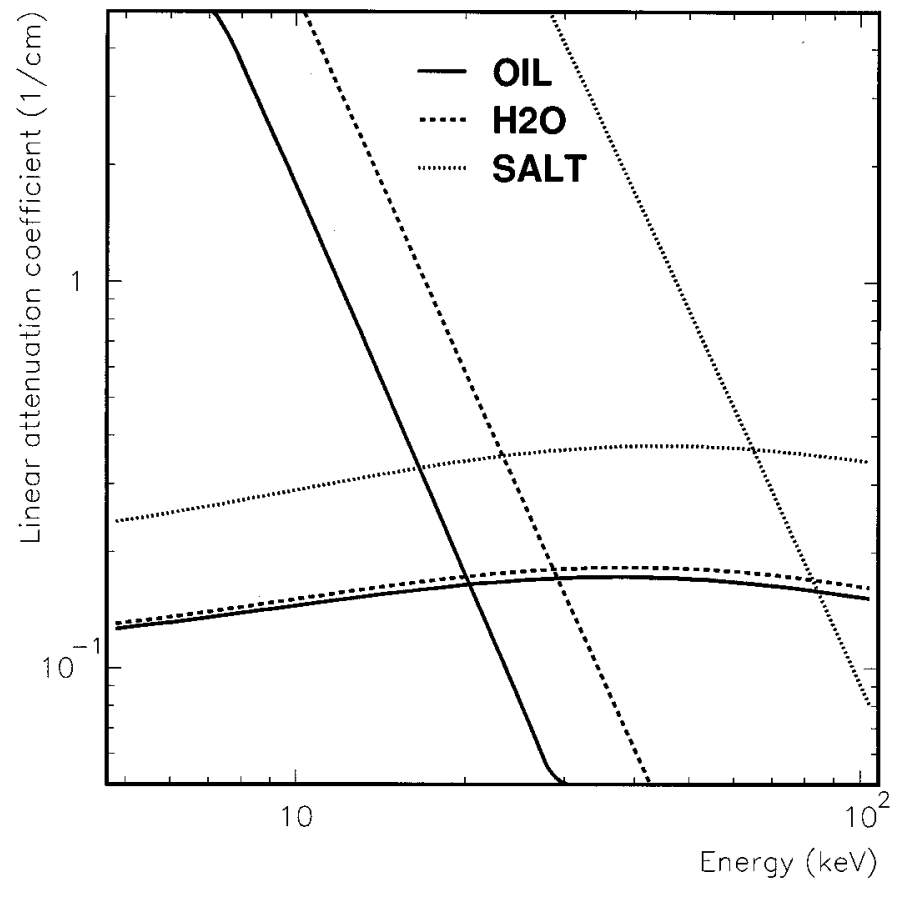

Fig. 4. Linear attenuation coefficients due to photoeffect, relative to the water attenuation coefficient, and normalized to $100 \%$ at $50 \mathrm{keV}$.

Increasing the number of X-ray energies will result in an overdetermined set of (12) and in a more accurate $P$ and $Q$, but it does not allow for more parameters to be derived.

\section{B. Using Real Attenuation Coefficients}

In real life, small influences of the atomic number of the material on the functions $\pi(E)$ and $\gamma(E)$ do exist. This means that

$$
\begin{aligned}
& \pi_{\mathrm{H}_{2} \mathrm{O}}(E) \approx \pi_{\mathrm{o}}(E) \approx \pi_{\mathrm{s}}(E) \\
& \gamma_{\mathrm{H}_{2} \mathrm{O}}(E) \approx \gamma_{\mathrm{o}}(E) \approx \gamma_{\mathrm{s}}(E) .
\end{aligned}
$$

This is shown in Figs. 3 and 4. In these figures, the attenuation coefficients of oil, gas, and salt are shown relative to the water attenuation coefficient and normalized to one at $50 \mathrm{keV}$. The 
TABLE I

DISTURBANCE FACTOR $\delta I / I$ OF THE INTENSITY OF ONE X-RAY LINE INTENSITY, WHICH RESULTS IN AN OIL FRACTION DEVIATION OF 0.01. THE OIL AND WATER FRACTIONS WERE 0.3 AND 0.6, RESPECTIVELY; THE SALINITY WAS $100 \mathrm{~kg} / \mathrm{m}^{3}$

\begin{tabular}{c|c|c|c}
\hline $\begin{array}{c}\text { Number of } \\
\text { energies }\end{array}$ & $\begin{array}{c}\text { Lowest } \\
\text { X-ray } \\
\text { energy } \\
{[\mathrm{keV}]}\end{array}$ & $\begin{array}{c}\text { Pipe } \\
\text { diameter } \\
{[\mathrm{cm}]}\end{array}$ & $\delta I / I$ \\
\hline \hline & 20 & 5 & 1.0002 \\
4 & 17 & 4 & 1.0004 \\
4 & 17 & 4 & 1.0004 \\
5 & 17 & 4 & 1.0008 \\
6 & 16 & 3 & 1.0009 \\
6 & 15 & 3 & 1.0012 \\
6 & & & \\
\hline
\end{tabular}

data are extracted from the GEANT simulation program [5]. The curves for gas and for oil are very similar and coincide in the figures. The curves in fact represent

$$
\begin{aligned}
\frac{\left(\frac{\pi_{\circ}(E)}{\pi_{\mathrm{H}_{2} \mathrm{O}}(E)}\right)}{\left(\frac{\pi_{\circ}(50 \mathrm{keV})}{\pi_{\mathrm{H}_{2}} \mathrm{O}(50 \mathrm{keV})}\right)}, \frac{\left(\frac{\pi_{\mathrm{s}}(E)}{\pi_{\mathrm{H}_{2} \mathrm{O}}(E)}\right)}{\left(\frac{\pi_{\mathrm{s}}(50 \mathrm{keV})}{\pi_{\mathrm{H}_{2} \mathrm{O}}(50 \mathrm{keV})}\right)}, \\
\frac{\left(\frac{\gamma_{\mathrm{s}}(E)}{\gamma_{\mathrm{H}_{2} \mathrm{O}}(E)}\right)}{\left(\frac{\gamma_{\mathrm{s}}(50 \mathrm{keV})}{\gamma_{\mathrm{H}_{2} \mathrm{O}}(50 \mathrm{keV})}\right)}, \text { etc. }
\end{aligned}
$$

The greater part of these curves lies around one, meaning only small shape differences. To increase the contrast between the various components, it is clearly necessary to move to much lower photon energies. However, this places significant limitations upon the fluid path length, transmission window characteristics, etc., that may be employed in any practical measurement system.

With the use of these "real" attenuation coefficients, the condition of Section III, which leads to (4), is slightly violated, and the possible determination of additional component fractions might arise.

1) Required Accuracy of Measurement: The possibility of the analysis of additional fractions, as mentioned above, has been investigated numerically. Assuming an input X-ray line spectrum with a certain number of infinitely narrow lines, theoretical "measured" line intensities were calculated. In a real flow meter, the mixture composition is determined by measuring an $\mathrm{X}$-ray spectrum and then extracting the intensities of the X-ray lines from this spectrum via some fit procedure. These intensities will thus come with some error or uncertainty. In the numerical case, also an uncertainty was imposed on the theoretical line intensities.

Next, the equation system (9) was solved, using the theoretical line intensities and accepting the salinity as a parameter to be determined. We found that indeed the salinity in addition to the absorber thicknesses could be analyzed when we did apply an uncertainty of zero. But with already small uncertainties in $I_{j}$ applied, the system of (9) becomes poorly conditioned such that large errors in the resulting salinity and thus in the absorber thicknesses $t_{i}$ may result.

These findings are illustrated in Table I. For the oil, water, gas, salinity combination of, respectively, 0.3, 0.6, 0.1, and 100 $\mathrm{kg} / \mathrm{m}^{3}$, and for a number of pipe diameters and number of energies used in the analysis, the table shows the disturbance factor $\delta I / I$, which, when applied to the intensity of one X-ray line, results in a $t_{\mathrm{o}}$ of 0.31 or 0.29 , and so in a deviation of 0.01 .

The energies were chosen within the interval [lowest energy: $60 \mathrm{keV}]$ to obtain a best result. We found that the results depended on the specific set of attenuation coefficients used, and also on the specific oil, water, gas, salinity combination. In general, the results indicated that an uncertainty in a line intensity of one part in $10^{3}$ causes an error in the $t_{\mathrm{o}}$ on the order of a few times 0.01; and so on the order of $10 \%$ in the case shown in Table I. Including the small Rayleigh effect did not relax this accuracy demand.

One can conclude from the values in the table that this accuracy demand is somewhat relaxed when going to lower X-ray energies and smaller pipe diameters. This is in agreement with Figs. 3 and 4, which also show an increasing violation of (4) toward lower energies.

In general, the mentioned measurement uncertainty in the $\mathrm{X}$-ray line intensity has a statistical and a systematic part. The statistical error originates from counting statistics, while the systematic error is caused by uncertainties and/or fluctuations in the system response function and the $\mathrm{X}$-ray spectrum shape. Of course the combination of both errors must meet the criterion stated above. Such errors will be the limiting factor in real-life situations.

While the statistical uncertainty can in principle be made arbitrarily small by increasing the radiation intensity, this is not true for the systematic uncertainty. But also a statistical uncertainty of $10^{-3}$ requires a number of counts of about $10^{6}$. When a counting time of $1 \mathrm{~s}$ is used to ensure a constant flow during the measurement, it will be clear that the detector system must be able to handle extreme high count rates.

The aforementioned errors apply to the absorption layer thicknesses inside the radiation beam, the $t_{i} \mathrm{~s}$. In translating these thicknesses to volume fractions, additional errors will be introduced when the mixture is not homogeneous or when the mixture composition is changing during the measurement.

\section{CONCLUSION}

$\mathrm{X}$-ray transmission is now established as a suitable technique for the analysis of oil, water and gas mixtures. At least two X-ray energies are needed for this purpose. Additional energies may give more accurate results but do not automatically allow the extraction of an additional component fraction.

To first order, it has been shown that the number of determinable parameters is related to the number of physical processes underlying the absorption, limiting that number to three. The derivation of more than three component fractions relies on small second-order differences between the absorption coefficients of the components involved. 
At the X-ray energies employed in typical oil-industry applications, the measurement accuracy required appears too large for practical implementation. The use of much lower photon energies relaxes the accuracy requirement but introduces other technical constraints upon any practical instrument.

\section{REFERENCES}

[1] S. Nuland, K. Skarsvåg, G. Sæther, and P. Fuchs, "Phase fractions in three-phase gas-oil-water flow," presented at the 5th Int. Conf. Multiphase Production, June 1991.
[2] M. D. Rebgetz, J. S. Watts, and H. W. Zastawny, "Determination of the volume fractions of oil, water and gas by dual energy gamma-ray transmission,” Int. J. Radiat. Appl. Instrum., pt. E, vol. 5, no. 4, 1991.

[3] A. M. Scheers and W. F. J. Slijkerman, "Multiphase flow metering using multiple energy gamma ray absorption (MEGRA) composition measurement," in Proc. SPE Annual Technical Conf., Oct. 1996.

[4] Z. I. Kolar, Optimization of a Dual Energy $\gamma$-or/and X-Ray Absorption System for Measuring Water-Oil Gas Volume Fractions, Delft, The Netherlands: Delft Univ. of Technology, Interfaculty Reactor Inst., 1991.

[5] R. Brun and F. Carminati, "GEANT detector description and simulation tool," in CERN Program Library Long Writeup W5013, 1994. 